\title{
Effect of Gamma Irradiation on the Structural and Electrical Properties of $\mathrm{Co}_{0.5} \mathrm{Zn}_{0.5} \mathrm{Ce}_{\mathrm{y}} \mathrm{Fe}_{2-\mathrm{y}} \mathrm{O}_{4}$
}

\author{
E. Ateia \\ Physics Department, Faculty of Science, Cairo University, Giza, Egypt
}

The effect of $\gamma$-irradiation on the structural and the electrical properties of rare earth ferrite of the general formula $\mathrm{Co}_{0.5} \mathrm{Zn}_{0.5} \mathrm{Ce}_{y} \mathrm{Fe}_{2-y} \mathrm{O}_{4}(0.0 \leq y \leq 0.2)$ is discussed. X-ray diffraction pattern of irradiated and un- irradiated samples showed the formation of cubic spinel structure. The obtained data show that the electrical conductivity $\ln \sigma$ as well as the dielectric constant $\varepsilon^{\prime}$ are highly dependent on both Ce ion concentration and radiation dose. The number of ferrous ion at octahedral sites play a dominant role in the change of the crystal size due to irradiation effect. All samples exhibit a change in slope of the conductivity versus inverse temperature giving two regions with different conduction mechanisms.

\section{Introduction:}

Ferrites continue to be very attractive materials for technological applications due to their combined properties as magnetic conductors (ferrimagnetic) and electric insulators. Polycrystalline ferrite, which have applications ranging from microwave frequencies to radio frequencies are very good dielectric materials [1].Magnetic therapy rare earth ferrite products studies have shown that Rare Earth Ferrite Products may be effective for the relief of acute and chronic pain or discomfort due to various injuries and ailments [2].

Co-Zn ferrite are quit important in the field of microwave industry, their usage is influenced by their physical and chemical properties which is in turn influenced by several factors such as method and conditions of preparations as well as the amount and type of additives[3]. Interaction of radiant energy with matter, especially $\gamma$ radiation, is an extremely important from the view point of theory and practice. When radiant energy acts on the materials, their electrical properties may change and new electrical phenomenon may be developed [4].

E-mail: drebtesam2000@yahoo.com 
The present investigation was carried out in an attempt to throw light on the effect of ionizing radiation and Ce ion concentration on the structure and electrical properties of Co-Zn Ce ferrite system.

\section{Experimental :}

The rare earth ferrite samples of general formula $\mathrm{Co}_{x} \mathrm{Zn}_{x} \mathrm{Ce}_{y} \mathrm{Fe}_{2-y} \mathrm{O}_{4}$ $(0.0 \leq y \leq 0.2) x=0.5$ were prepared by usual standard ceramic technique[5] using analar grade oxides (BDH). The pre sintering was carried out at $850{ }^{\circ} \mathrm{C}$ for 30 hours using lenton furnace type UAF 16/5 (England). The samples were compressed into pellet form using hydraulic press with pressure of $5 \times 10^{8} \mathrm{~N} / \mathrm{m}^{2}$. The final sintering was carried out at $1200{ }^{\circ} \mathrm{C}$ for $30 \mathrm{hrs}$ and then cooled to room temperature with the same rate as that of heating $\left(4^{\circ} \mathrm{C} / \mathrm{min}\right)$. The pellets were well polished and the two surfaces of each pellet were coated with silver paste and checked for good conduction. X-ray diffraction patterns were carried by Diano X-ray diffractometer with $\mathrm{Co} \mathrm{K} \alpha$ radiation to characterize the formation of the sample structures.

Co Zn Ce ferrite tablets were exposed to $\mathrm{Co}^{60} \gamma$-rays at different doses (100 krad, $500 \mathrm{krad}$, and 5Mrad) at the Technological Research Center of radiation (Nasr city, Cairo). All measurements were performed before as well as after irradiation. The surface of the sample was imaged by a scanning electron microscope (SEM) model JSM-T330-Scanning Microscope. The micrographs with a suitable magnification depending on the samples were taken and the average grain size calculated.

The real part of the dielectric constant $\left(\varepsilon^{\prime}\right)$ was calculated from the relation:

$$
\varepsilon^{\prime}=\frac{C L}{\varepsilon \circ A}
$$

where $C(F)$ and $L(\mathrm{~cm})$ are the capacitance and thickness of the samples respectively. $\varepsilon_{0}(\mathrm{~F} / \mathrm{cm})$ is the permittivity of free space and $\mathrm{A}\left(\mathrm{cm}^{2}\right)$ is the area of the samples. While the ac conductivity $\sigma\left(\mathrm{ohm}^{-1} \mathrm{~cm}^{-1}\right)$ was calculated from the relation $\sigma=\frac{L}{A R}$ where $\mathrm{R}(\mathrm{ohm})$ is the resistance of the samples. $\varepsilon$ and $\sigma$ were recorded at different temperatures as a function of frequency using RLC bridge model HIOKI 3530 (Japan). 


\section{Results and Discussion:}

\subsection{X- Ray Analysis:}

Figure (1) represents the diffraction patterns for $\mathrm{Co}_{0.5} \mathrm{Zn}_{0.5} \mathrm{Ce}_{\mathrm{y}} \mathrm{Fe}_{2-\mathrm{y}} \mathrm{O}_{4}$ $\mathrm{y}=0.0,0.05,0.20$ before and after radiation. The diffraction patterns shows that, all samples exhibit a cubic spinel structure with appearance of some extra peaks designated as secondary phases.

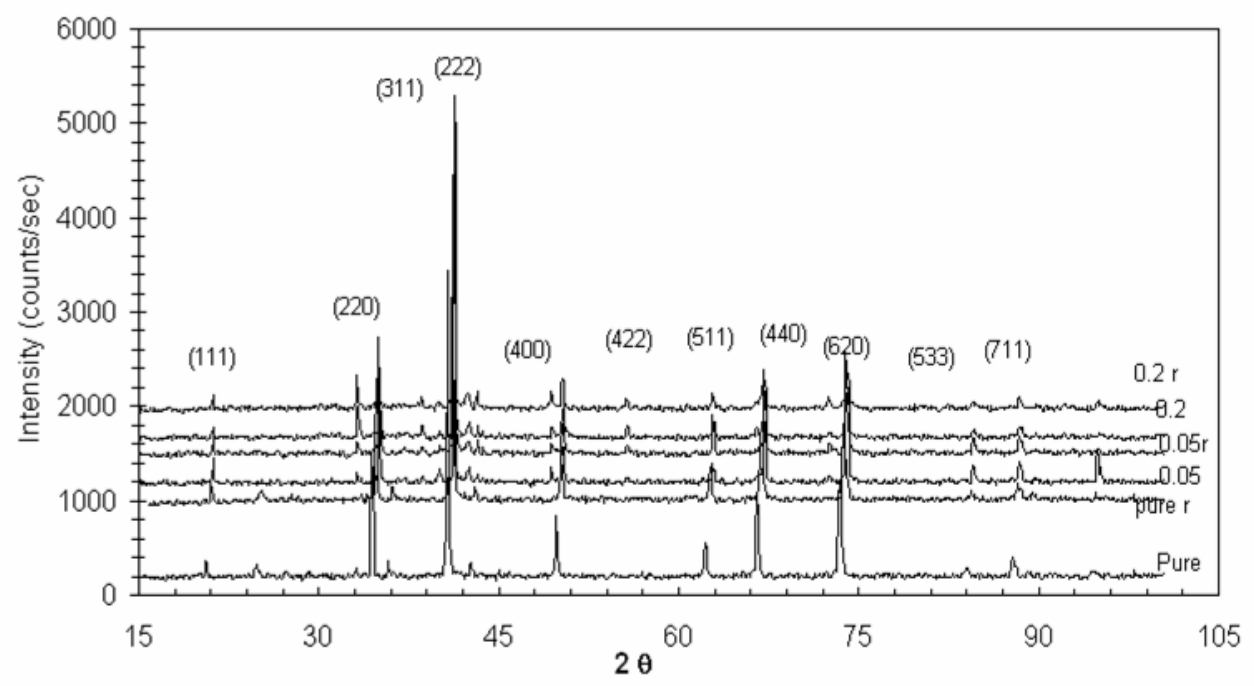

Fig.(1): X-ray diffractograms for radiated and unradiated $\mathrm{Co}_{0.5} \mathrm{Zn}_{0.5} \mathrm{Ce}_{\mathrm{y}} \mathrm{Fe}_{2-\mathrm{y}} \mathrm{O}_{4}$

The calculated values of the lattice parameter (a) for the investigated samples verified that $\mathrm{Ce}^{3+}$ doesn't occupy the tetrahedral or octahedral sites $[6,7]$ but forms aggregates on the grain boundaries, or in sub-boundaries, composed of an arrays of dislocations. Such sub-boundaries permit the existence of coincidence sites, in the grain boundaries, ie. Ce ions assume positions that tend to minimize the local elastic strain.

The correlation between the ionic radii and the theoretical lattice constant $\left(\mathrm{a}_{\mathrm{th}}\right)$ is calculated using [8]:

$$
a_{\text {th }}=\frac{8}{3 \sqrt{3}}\left[\left(r_{A}+R_{o}\right)+\sqrt{3}\left(r_{B}+R_{o}\right)\right]
$$

where $R_{0}$ is the radius of the oxygen ion $(0.138 \mathrm{~nm})$ [9], $r_{A}$ and $r_{B}$ are the ionic radii of tetrahedral(A) and octahedral (B) sites respectively. In order to calculate, $r_{A}$ and $r_{B}$ it is necessary to know the cation distribution for a given 
system (Co-Zn- Ce ferrite). In general $\mathrm{Zn}$ ferrite is normal ferrite, Co is an inverse ferrite[10] and $\mathrm{Fe}^{3+}$ ions are distributed between the two sites [11,12]. Therefore Co- Zn- Ce ferrite is a mixed type with interesting properties. Accordingly, the cation distribution of the investigated samples can be expressed in a general proposed form as follows,

$$
\left(\mathrm{Zn}_{\mathrm{x}}{ }^{2+} \mathrm{Fe}_{1-\mathrm{x}}{ }^{3+}\right) \quad\left[\mathrm{Co}_{1-\mathrm{x}}{ }^{2+} \mathrm{Fe}_{1+\mathrm{x}}{ }^{3+}\right]
$$

where the brackets ( $)$ and [ ] indicate A and B sites respectively. In our case $\mathrm{x}=0.5$ then $\mathrm{r}_{\mathrm{A}}$ and $\mathrm{r}_{\mathrm{B}}$ can be calculated by using the following equations:

$$
\begin{aligned}
& \mathrm{r}_{\mathrm{A}}=0.5 \mathrm{r}_{\mathrm{Zn} 2+}+0.5 \mathrm{r}_{\mathrm{Fe} 3+} \\
& \mathrm{r}_{\mathrm{B}}=1 / 2 \quad\left[0.5 \mathrm{r}_{\mathrm{Co} 2+}+1.5 \mathrm{r}_{\mathrm{Fe} 3+}\right]
\end{aligned}
$$

Using the following ionic radii $\mathrm{Zn}^{2+}\left(0.600{ }^{\circ} \mathrm{A}\right), \mathrm{Co}^{2+}\left(0.610^{\circ} \mathrm{A}\right)$ and $\mathrm{Fe}^{3+}(0.4900 \AA$ and $0.6450 \AA)$ depending on its coordination number $[5,13]$, $\mathrm{a}_{\mathrm{th}}$ can be calculated and tabulated in Table (1). As shown from the table $a_{\text {th }}$ is calculated for pure sample only; because Ce ion doesn't enter into the spinel structure but cause redistribution of $\mathrm{Co}, \mathrm{Zn}$ and $\mathrm{Fe}$ and it is difficult to expect cation distribution in this case.

The theoretical oxygen positional parameter $(\mathrm{u})$ is given in Table (1) which is nearly the same as that obtained for spinel ferrites samples[14] The oxygen positional parameter $(\mathrm{u})$ is given by the following relation $[15,16]$,

$$
\mathrm{u}=\left[\left(\mathrm{r}_{\mathrm{A}}+\mathrm{R}_{\mathrm{o}}\right) \frac{1}{\sqrt{3} a}+\frac{1}{4}\right]
$$

Table (1): Theoretical, experimental lattice constant and oxygen positional parameter.

\begin{tabular}{|c||c|c|c||}
\hline $\mathrm{y}$ & $\begin{array}{c}\text { Theoretical lattice } \\
\text { constant, } \mathrm{a}_{\mathrm{th}}(\AA)\end{array}$ & $\begin{array}{c}\text { Experimental lattice } \\
\text { constant, } \mathrm{a}_{\exp }(\AA)\end{array}$ & $\mathrm{u}_{\text {exp. }}(\AA)$ \\
\hline 0.00 & 8.340 & 8.527 & 0.3855 \\
\hline 0.05 & - & 8.416 & - \\
\hline 0.20 & - & 8.445 & - \\
\hline
\end{tabular}

The crystallite size and micro strain of the investigated samples are calculated by using Win fit program [17] and tabulated in Table (2). From the table it is clear that, the crystallite size for un-irradiated samples is greater than irradiated one, while the estimated micro strain values for irradiated samples is greater than un-irradiated one. This can be attributed to strong effect of $\gamma$ irradiation on the structure of the investigated samples. 
Table (2): Values of crystallite size and micro strain of irradiated and unirradiated $\mathrm{Co}_{0.5} \mathrm{Zn}_{0.5} \mathrm{Ce}_{\mathrm{y}} \mathrm{Fe}_{2-\mathrm{y}} \mathrm{O}_{4}$

\begin{tabular}{|c||c|c|c|c||}
\hline \hline \multirow{2}{*}{$\mathrm{y}$} & \multicolumn{2}{|c|}{ Un-irradiated } & \multicolumn{2}{c|}{ Irradiated } \\
\cline { 2 - 5 } & $\begin{array}{c}\text { Crystallite size } \\
(\AA)\end{array}$ & Microstrain & $\begin{array}{c}\text { Crystallite size } \\
(\AA)\end{array}$ & Microstrain \\
\hline \hline 0.05 & 1654 & 0.00024 & 816 & 0.00185 \\
\hline 0.20 & 1362 & 0.00022 & 717 & 0.00179 \\
\hline
\end{tabular}

The scanning electron micro graphs of the irradiated and un- irradiated samples for $\mathrm{y}=0.0,0.05$, and 0.2 are given in Fig (2:a-e). All samples were sintered at $1200^{\circ} \mathrm{C}$ with heating rate $4^{\circ} \mathrm{C} / \mathrm{min}$ for $30 \mathrm{hrs}$. The grain size for radiated and irradiated samples were calculated and reported as a function of $\mathrm{Ce}$ ion concentration in Table (3). The data in Table 3 shows the decrease in grain size for radiated sample with respect to unradiated one. Also grain size decreases with increasing Ce ion concentration, which agree well with the calculated crystallite size.
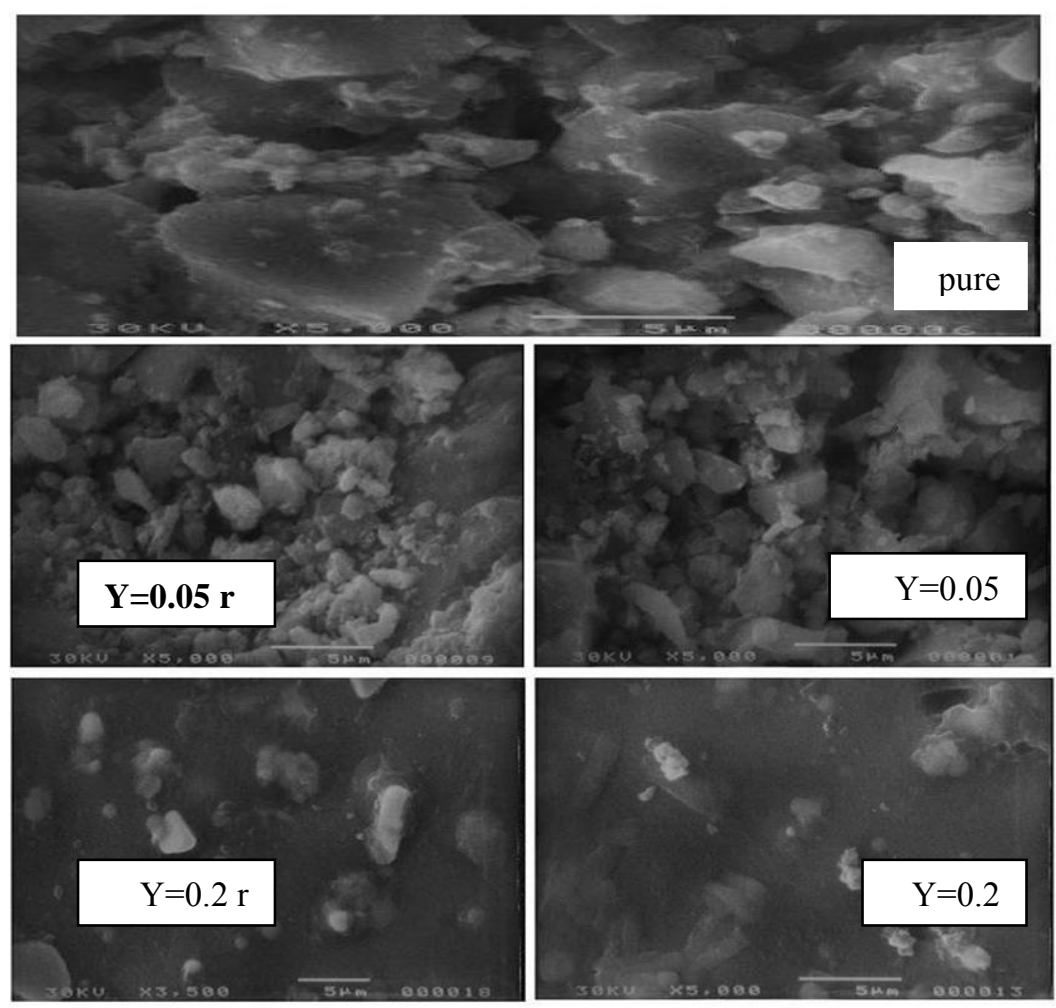

Fig.(2): The scanning electron micrographs for un irradiated and irradiated samples. 
Table (3): Effect of Ce ion concentration and radiation doses on the grain size.

\begin{tabular}{|c||c|c|}
\hline \multirow{2}{*}{$\mathrm{y}$} & \multicolumn{2}{|c|}{ Grain size $(\mu \mathrm{m})$} \\
\cline { 2 - 3 } & Un-irradiated & Irradiated \\
\hline 0.00 & 2.66 & - \\
\hline 0.05 & 2.190 & 1.678 \\
\hline 0.20 & 1.643 & 1.545 \\
\hline
\end{tabular}

\subsection{Electrical Properties}

Fig. (3:a-c) correlates the variation of the dielectric constant $\left(\varepsilon^{\prime}\right)$ with the absolute temperature at different frequencies for the un-irradiated and irradiated $\mathrm{Co}_{0.5} \mathrm{Zn}_{0.5} \mathrm{Ce}_{\mathrm{y}} \mathrm{Fe}_{2-\mathrm{y}} \mathrm{O}_{4} ; \mathrm{y}=0.05$ with different doses of $0.0,100 \mathrm{krad}$ and 5Mrad.
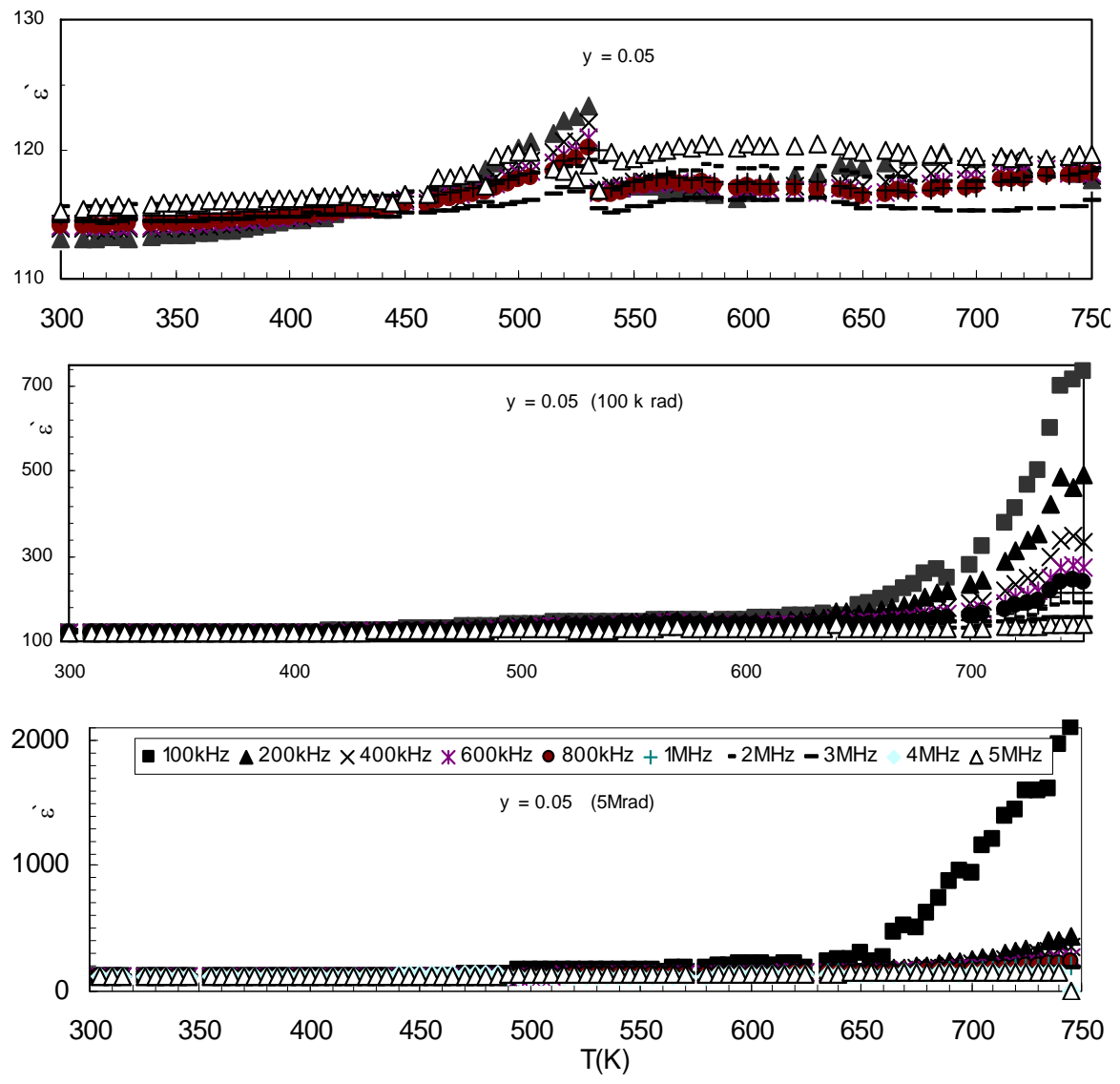
Fig.(3): Variation of the real part of the dielectric constant with the absolute temperature at different frequencies for irradiated and un-irradiated ferrite samples.

The general trend of all samples is the increase in $\left(\varepsilon^{\prime}\right)$ with increasing temperature, but for un-irradiated sample $\left(\varepsilon^{\prime}\right)$ is nearly constant with a change in the temperature.

However, for irradiated one, the dielectric constant $\left(\varepsilon^{\prime}\right)$ shows an explicit increase with temperature. This can be divided into two regions. In the lower temperature region up to $650 \mathrm{~K},\left(\varepsilon^{\prime}\right)$ increases slightly with the temperature because the thermal energy given to the sample is not sufficient enough to free the localized dipoles to be oriented in the field direction. Above $\mathrm{T}=650 \mathrm{~K}\left(\varepsilon^{\prime}\right)$ increases more clearly but with different rates depending on the irradiation doses. This increase in $\left(\varepsilon^{\prime}\right)$ is due to the large number of dipoles that become free such high thermal energy and the field aligned them in its direction. The higher value of $\left(\varepsilon^{\prime}\right)$ is obtained for irradiated samples, this can be explained in a view of interaction of $\gamma$ - rays with the matter. This interaction is summarized in two steps: the first one is the increase in hopping rate caused from depressing the jump length with the result of more interaction between $\mathrm{Fe}^{3+} \leftrightarrow \mathrm{Fe}^{2+}$, and $\mathrm{Co}^{3+} \leftrightarrow \mathrm{Co}^{2+}$. The jumping electrons oriented in the field direction and, consequently, giving rise to $\varepsilon^{\prime}$. The production of $\mathrm{Fe}^{2+}$ after irradiation was confirmed by Mousa et al. [18]. Second step is the generation of some vacancies at different depths which act as trapping centers. The liberation of charge carriers from these trapping centers needs different energies. This seems to be the main reason for highest value of $\varepsilon^{\prime}$ in the case of $5 \mathrm{Mrad}$ irradiated sample.

The decreasing of $\left(\varepsilon^{\prime}\right)$ with increasing frequency is a general trend of the investigated samples. A similar variation has been reported by Josyulu and Sobhanadri [6] This decrease is due to the electron exchange do not follow the frequency of alternating electric field beyond a certain frequency [19].

The variation of the dielectric constant $\left(\varepsilon^{\prime}\right)$ with $\mathrm{Ce}$ ion concentration $0.0 \leq \mathrm{y} \leq 0.2$ at selected frequency $(100 \mathrm{KHz}, 1 \mathrm{MHz})$ and temperature $\left(600{ }^{\circ} \mathrm{K}\right)$ for $\mathrm{Co}_{0.5} \mathrm{Zn}_{0.5} \mathrm{Ce}_{\mathrm{y}} \mathrm{Fe}_{2-\mathrm{y}} \mathrm{O}_{4}$ is shown in Fig. (4). It can be seen from the figure that, $\varepsilon$ ' increases with increasing Ce ion concentration up to 0.175 then decreases, this can be explained in view of behavior of $\mathrm{Ce}$ ions. That is $\mathrm{Ce}^{3+}$ ion is a non typical rare earth element having a broader width of $4 \mathrm{f}$ band, consequently giving rise to a strong hybridization of $4 \mathrm{f}$ state with $3 \mathrm{~d}$ band [20]. The valence fluctuation between $\mathrm{Ce}^{3+}$ and $\mathrm{Ce}^{4+}$ ions with the above factors are the main reasons for the highest value of $\left(\varepsilon^{\prime}\right)$. The decrease after 0.175 is due to 
formation of insulating intergranular layers which impeds the oxidation of $\mathrm{Fe}^{2+}$ ions inside grains during cooling of the samples [21].

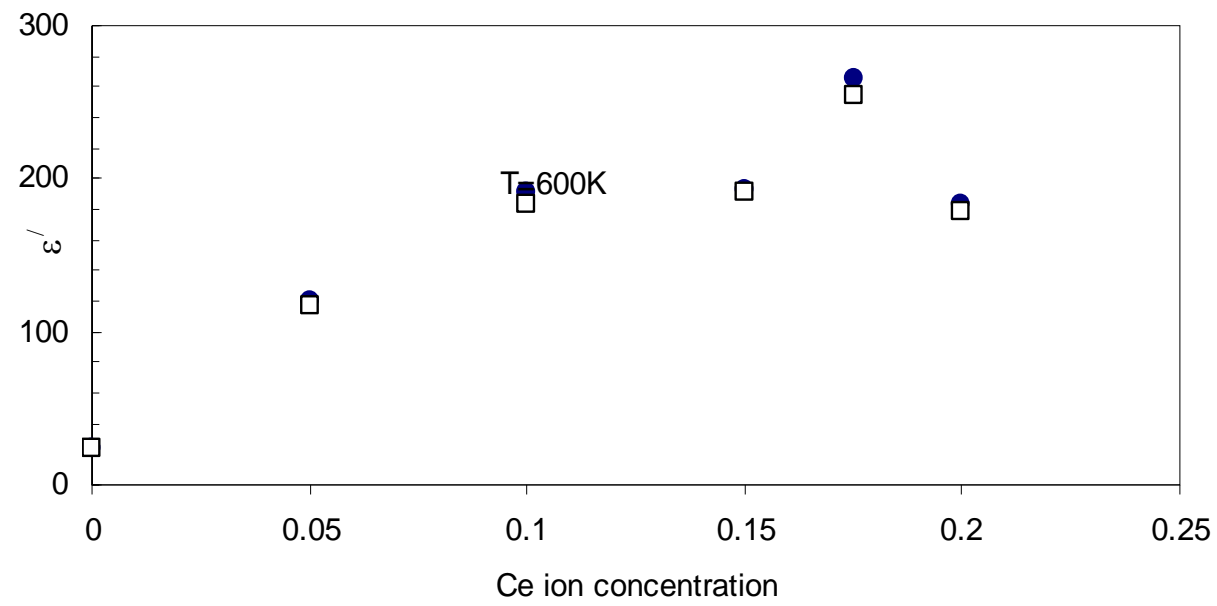

Fig. (4): Effect of Ce ion concentration on the real part of the dielectric constant

Figure (5) is a typical curves correlates the dielectric loss factor $\varepsilon^{\prime \prime}$ and the absolute temperature at different frequencies for the samples of the general formula $\mathrm{Co}_{0.5} \mathrm{Zn}_{0.5} \mathrm{Ce}_{\mathrm{y}} \mathrm{Fe}_{2-\mathrm{y}} \mathrm{O}_{4} ; \mathrm{y}=0.2$. The figure shows a continuous increase in $\varepsilon^{\prime \prime}$ with the temperature until reaching a maximum. The decrease in $\varepsilon^{\prime \prime}$ with increasing frequency agree well with Deby's type relaxation process [22]. The occurrence of loss peaks can be discussed in view of the strong correlation between the conduction mechanism and the dielectric behavior for the spinel ferrites as Iwauchi[19] pointed out. The conduction mechanism in the investigated samples is explained in terms of the hopping conduction process which occurs among the octahedral B sites. In this case a maximum of $\varepsilon^{\prime \prime}$ is observed when the hopping frequency becomes equal to the external electric field frequency[23]. However the increase in $\varepsilon^{\prime \prime}$ with the temperature is due to the relaxation of the dipole molecules coupled with the resulting drop in relaxation time. This in turn exerts a double effect on the dielectric loss on one hand the friction between the dipoles will be increased and on the other hand the energy required to overcome the internal mechanical friction of the medium will be decreased when the dipoles rotate through a unit angle . 

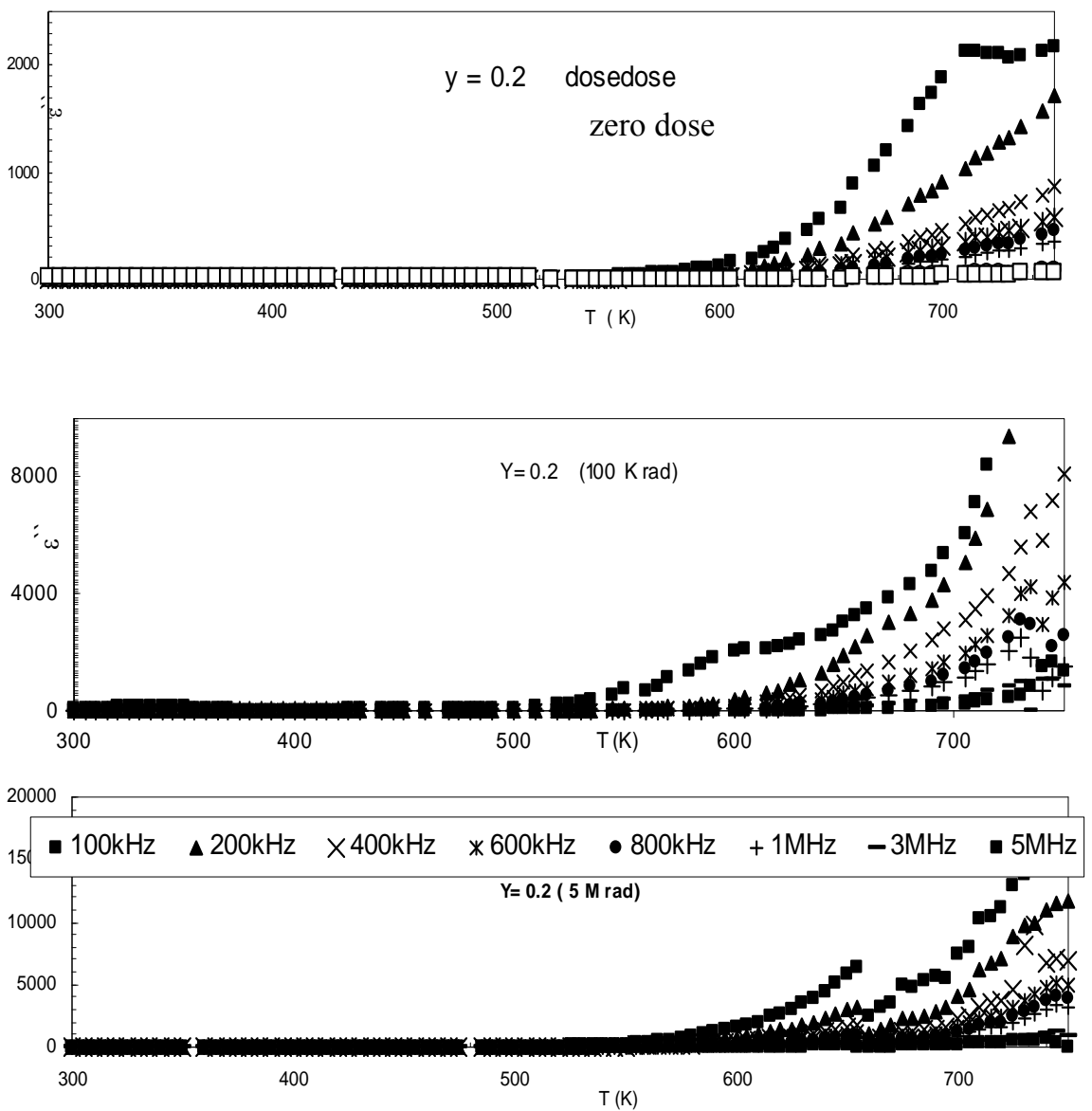

Fig. (5): Variation of dielectric loss with the absolute temperature at different Frequencies for irradiated and un irradiated $\mathrm{Ce}$ doped $\mathrm{Co}-\mathrm{Zn}$ ferrite.

Figure (6) shows the dependence of the ac conductivity $(\ln \sigma)$ for the investigated samples of the general formula $\mathrm{Co}_{0.5} \mathrm{Zn}_{0.5} \mathrm{Ce}_{\mathrm{y}} \mathrm{Fe}_{2-\mathrm{y}} \mathrm{O}_{4} ; \mathrm{y}=0.2$ at different frequencies with different irradiation doses $(0.0,100 \mathrm{krad}, 5 \mathrm{Mrad})$. The obtained data obeys the well known Arrhenious relation[10]. $\sigma=\sigma_{0} \exp$ $(-\mathrm{E} / \mathrm{kT})$, where $\sigma_{\mathrm{o}}$ is constant, $\mathrm{k}, \mathrm{E}$ and $\mathrm{T}$ are the Boltzmann's constant, activation energy and the absolute temperature respectively. Increasing temperature leads to an increase in $\sigma$ which is the normal behavior of the semi conducting materials two conduction mechanisms are expected here, the first 
being the hopping mechanism below the transition temperature, where the conductivity is mainly frequency and temperature dependent [24]. While the second mechanism above the transition temperature which is temperature dependent and frequency independent, is related to the drift mobility of the thermally activated electrons and not to thermally creation of the charge carriers. The valance exchange between $\mathrm{Fe}^{3+} \Leftrightarrow \mathrm{Fe}^{2+}$ and the hole hopping between $\mathrm{Co}^{2+} \Leftrightarrow \mathrm{Co}^{3+}$ at $\mathrm{B}$ sites are the main source of hopping mechanism in our case. From a closer look of Fig. 6 it is clear that the conductivity increases as the $\gamma$ dose increases. This increases in $\sigma$ can be attributed to the increase in the ratio of $\mathrm{Fe}^{3+} / \mathrm{Fe}^{2+}$ and $\mathrm{Co}^{2+} / \mathrm{Co}^{3+}$ on the octahedral sites as a consequence of hopping reaction[25]. By comparing the transition temperature $\mathrm{T}_{\mathrm{c}}$ of the investigated samples $(0.0,100 \mathrm{krad}, 5 \mathrm{Mrad})$, one can find that, the transition temperature $T_{c}$ increases with increasing doses as a result of increasing the ordered region.
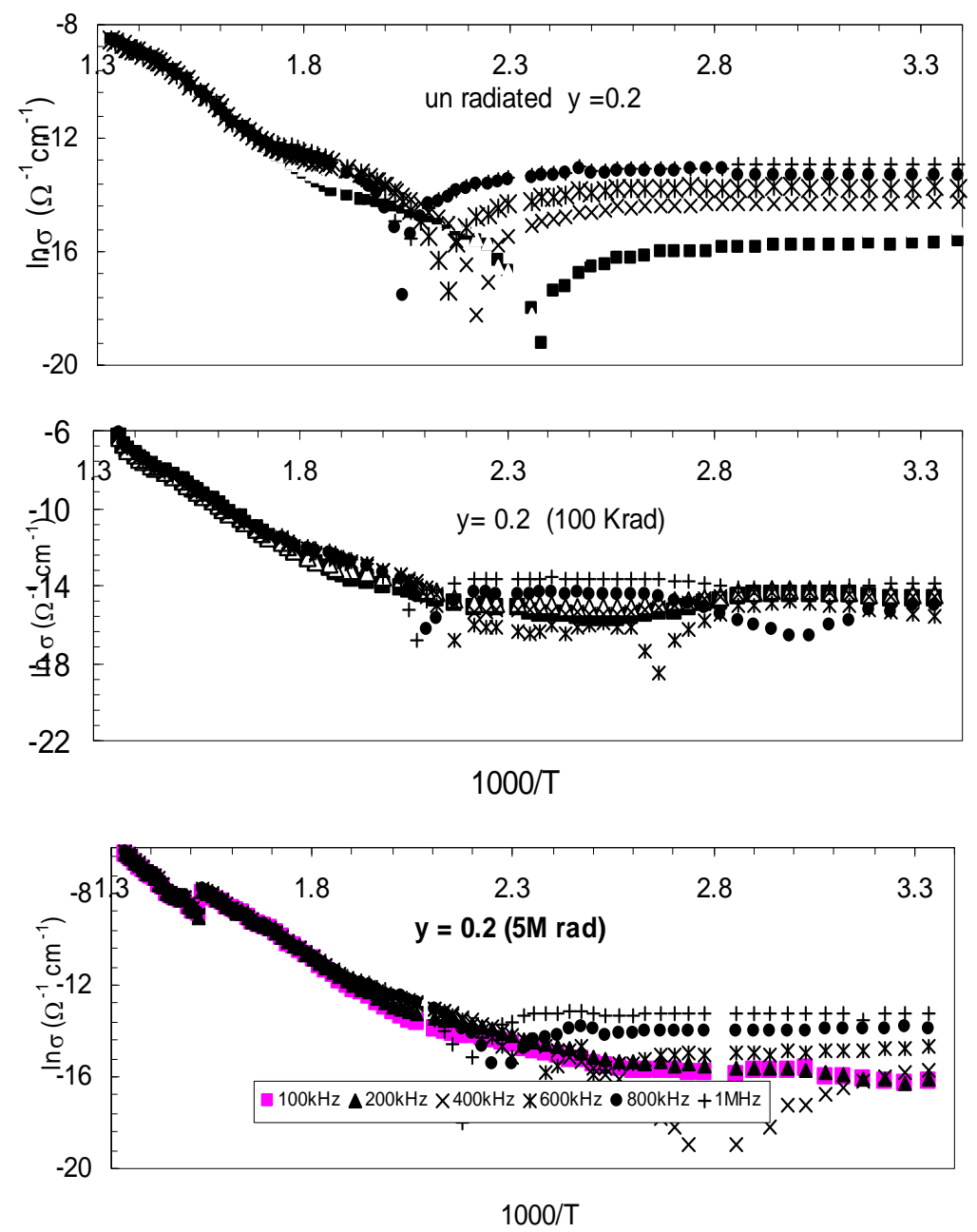
Fig.(6): Variation of ac conductivity ( $\ln \sigma$ ) with the reciprocal of absolute temperature $(1000 / T)$ for irradiated and un irradiated Co- $\mathrm{Zn}$ ferrite samples.

In other words, the arrangement of the magnetic dipoles takes place due to effect of the energy of $\gamma$-radiation on the expanse of paramagnetic region, though increasing transition temperature $\mathrm{T}_{\mathrm{c}}$.

\section{Conclusion:}

X-ray diffractograms assured the cubic spinel structure for all investigated samples, with the appearance of small peaks corresponding to the secondary phase due to the presence of $\mathrm{Ce}$ ions. The calculated crystallite sizes and the grain size as estimated from scanning electron micrographs are in good agreement, since both decrease with increasing $\mathrm{Ce}$ ion concentration and after radiation doses. The electrical properties of the investigated samples are highly dependent on the radiation doses. The decrease in $\varepsilon^{\prime}$ with increasing frequencies for all samples is attributed to the decrease in the polarization of the sample because the dipoles do not follow the field variation.

\section{References:}

1. J. Smit and J. Verweel, "Ferrites at radio frequencies", McGrow-Hill, New York, (1971).

2. J. M. Cadogan, Hong-Shuo Li, A. Margarian, J. B. Dunlop, D. H. Ryan, S. J. Collocott and R. L.Davis, J. Appl. Phys. 76, 6138 (1994)

3. N. Rezlescu and E. Rezlescu, J. Phys. Candens Matter. 6, 5707(1994).

4. B. Tareev, "Physics of Dielectric Materials", Mir Publisher, Moscow (1979).

5. R. Valenzuela, "Magnetic Ceramics", Cambridge University, (1994).

6. O. S.Josyulu, J. Sobhanadri, B. Viswanatham, Rev. Roum. Chim. 26 (5) 687, (1981).

7. E. Rezlescu, N. Rezlescu, P.D. Popa, L. Rezlescu, and C. Pasnicu, phys. stat. sol. (a), 162, 673(1997).

8. S. A. Mazen, M. H. Abdallah, B. A. Sabrah, and H.A.M. Hasem, phys. stat. sol. A, 134, 263 (1992).

9. R. D. Shanon, Acta Cryst. A32, 75(1976)

10. J. Smit, H. P. Wijn, "Ferrites", Cleaver- Hume press, London (1959).

11. B. Viswanathan, and V.R.K. Murthy "Ferrite Materials Science and Technology", Narosa Publishing House (1990).

12. N. Rezlescu, E. Rezlescu and C. Pasnicu and M. L. Craus, J. Phy. Condensed Matter 6,5707(1994). 
13. Periodic Table, SARGENT-WELCH, Scientific Company, 7300 Linder Avenue, Skokie, Illinois 60076, Catalog Number 5-18806.

14. E. J. W. Verwey, E.Z. Heilman, J. Chem. Phys. 15(4), 174 (1947)

15. O. A. Hemeda, M.Z. Said, and M. M. Barakat, J. Mag. and Mag. Mat., 224, 132 (2001)

16. K. J. Standley, "Oxide Magnetic Materials", Clarendon Press Oxford (1972).

17. S. Krumn; Acta Universitatis Carolinae Geologica 38, 253, (1994).

18. M. A. Mousa, A. M. Summan, M. A. Ahmed and A. M. Badawy, Thermochimica Acta, 144, 45 (1989).

19. K. Iwauchi, Jpn. J. Appl. Phys., 10, 1520 (1971)

20. N. Rezlescu, and E. Rezlescu, Solid State Communications, 88, 139 (1993)

21. N. Rezlescu, E. Rezlescu and C. Pasnicu and M. L. Craus, J. Phy. Condensed Matter, 6, 5707(1994)

22. P. Debye, "Polar Molecules", chemical catalogue Company, New York, (1929).

23. V. R. K. Murthy, J. Sobhanadri, phys. status solidi A, 36, K133 (1976)

24. M. Abdeen, J. Mag. and Mag. Mat. 185, 199 (1998).

25. A. Goldman "Modern Ferrite Technology", Marcel Dekker, New York (1993). 